1 Hacettepe Journal of Mathematics and Statistics

h Volume 45 (3) (2016), 649-661

\title{
Small supplements, weak supplements and proper classes
}

\author{
Rafail Alizade* ${ }^{*}$ Engin Büyükaşık $k^{\dagger}$ and Yılmaz Durğun ${ }^{\ddagger \S}$
}

\begin{abstract}
Let $\mathcal{S} \mathcal{S}$ denote the class of short exact sequences $\mathbb{E}: 0 \rightarrow A \stackrel{f}{\rightarrow} B \rightarrow$ $C \rightarrow 0$ of $R$-modules and $R$-module homomorphisms such that $f(A)$ has a small supplement in $B$ i.e. there exists a submodule $K$ of $M$ such that $f(A)+K=B$ and $f(A) \cap K$ is a small module. It is shown that, $\mathcal{S S}$ is a proper class over left hereditary rings. Moreover, in this case, the proper class $\mathcal{S} \mathcal{S}$ coincides with the smallest proper class containing the class of short exact sequences determined by weak supplement submodules. The homological objects, such as, $\mathcal{S} \mathcal{S}$-projective and $\mathcal{S} \mathcal{S}$ coinjective modules are investigated. In order to describe the class $\mathcal{S} \mathcal{S}$, we investigate small supplemented modules, i.e. the modules each of whose submodule has a small supplement. Besides proving some closure properties of small supplemented modules, we also give a complete characterization of these modules over Dedekind domains.
\end{abstract}

Keywords: Proper class of short exact sequences, weak supplement submodule, small module, small supplement submodule

2000 AMS Classification: 18G25, 16D10, 16E30, 13F05

Received: 15.07.2014 Accepted: 20.04.2015 Doi : 10.15672/HJMS.20164512507

*Yaşar University, Department of Mathematics, Üniversite Cad., No. 35-37, 35100, Bornova, İzmir Turkey, Email: rafail.alizade@yasar.edu.tr

†İzmir Institute of Technology, Department of Mathematics Gülbahçe köyü, 35430, Urla, Izmir, Turkey, Email: enginbuyukasik@iyte.edu.tr

${ }^{\ddagger}$ Bitlis Eren University, Department of Mathematics, 13000, Bitlis, Turkey, Email: ydurgun@beu.edu.tr

${ }^{\S}$ Corresponding Author. 


\section{Introduction}

All rings are associative with identity element and all modules are unitary left modules. We use the notation $E(M), \operatorname{Soc}(M), \operatorname{Rad}(M)$, for the injective hull, socle, radical of an $R$-module $M$ respectively. Let $M$ be any module and let $N$ and $K$ be submodules of M. $N$ is said to be small (or superfluous) in a module $M$, denoted as $N \ll M$ if $N+K=M$ implies $K=M$ for any submodule $K$ of $M . N$ is said to be a small module if $N$ is a small submodule of some $R$-module. $N$ is a small module if and only if $N$ is a small submodule of its injective envelope (see, [9]). A submodule $N$ of $M$ is called a supplement of $K$ in $M$ if $N$ is minimal with respect to the property $M=K+N$, equivalently, $M=K+N$ and $K \cap N \ll N$. A submodule $K$ of $M$ has a supplement in $M$ provided there exists a submodule $N$ of $M$ such that $N$ is a supplement of $K$ in $M$. A submodule $N$ of $\mathrm{M}$ has (is) a weak supplement $K$ in $M$ if $M=K+N$ and $K \cap N \ll M$. If every submodule of $M$ has a (weak) supplement in $M$, then $M$ is called (weakly) supplemented.

Proper classes were introduced by Buchsbaum in order to axiomatize conditions under which a class of short exact sequences of modules can be computed as Ext groups corresponding to a certain relative homology. Let $\mathbb{E}: 0 \rightarrow A \stackrel{f}{\rightarrow} B \rightarrow C \rightarrow 0$ be a short exact sequence. It is well-known that the class of short exact sequences $\mathbb{E}$ such that $\operatorname{Im}(f)$ is a supplement in $B$, respectively pure in $B$ is a proper class in the sense of Buchsbaum (see, $[7,20.7])$. However, many other analogous classes of short exact sequences of modules do not form a proper class. For example, the classes $\mathcal{S}$ mall, $\mathcal{S}$ or $\mathcal{W} \mathcal{S}$ i.e. the classes of short exact sequences $\mathbb{E}$ such that $\operatorname{Im} f$ small in $B$, has a supplement in $B$, or has a weak supplement in $B$, respectively, are not proper classes. But, in this case, one may consider the least proper class containing a given class of short exact sequences, that is, the intersection of all proper classes containing them. Recently, in [3], the authors shows that, the least proper classes containing the classes $\mathcal{S}$ mall, $\mathcal{S}$ or $\mathcal{W} \mathcal{S}$ coincide over hereditary rings. They obtained this proper class by natural extension of the class $\mathcal{W} \mathcal{S}$ and denoted it by $\overline{\mathcal{W S}}$.

At this point, the question which arises naturally is that, whether the class $\overline{\mathcal{W S}}$ can be described as a class of short exact sequences $\mathbb{E}$ such that $\operatorname{Im}(f)$ has a certain property in $B$. The answer of this question is affirmative over left hereditary rings. Over such rings the class $\overline{\mathcal{W S}}$ coincides with the class determined by small supplements.

The paper is organized as follows.

In section 3, weakening the notion of weak supplement we consider small supplement submodules. Namely, a submodule $N$ of a module $M$ has a small supplement in $M$ if there exists a submodule $K$ of $M$ such that $N+K=M$ and $N \cap K$ is a small module. Let $\mathcal{S} \mathcal{S}$ be the class of short exact sequences such that $\operatorname{Im}(f)$ has a small supplement in $B$. We prove that, $\mathcal{S} \mathcal{S}$ is a subgroup of Ext, and over a hereditary ring $\mathcal{S} \mathcal{S}$ is a proper class. Moreover, $\mathcal{S} \mathcal{S}$ coincides with the proper class $\overline{\mathcal{W S}}$.

In section 4 , we investigate $\mathcal{S} \mathcal{S}$-projective modules which are projective relative to short exact sequences that belong to $\mathcal{S} \mathcal{S}$. We show that an $R$-module $F$ is $\mathcal{S} \mathcal{S}$-projective if and only if $\operatorname{Ext}(F, S)=0$ for each small $R$-module $S$. Moreover, we prove that every $\mathcal{S} \mathcal{S}$-projective module is flat if $R$ is commutative $C$-ring (i.e. $\operatorname{Soc}(R / I) \neq 0$ for each essential proper left ideal $I$ ).

In section 5, we study on the properties of the modules whose submodules have small supplements. We call these modules small supplemented. Small supplemented modules are proper generalization of weakly supplemented modules. It is shown that, small supplemented modules are closed under submodules, factor modules, finite sums and extensions. An injective module is small supplemented if and only if it is weakly supplemented. 
In section 6, we characterize small supplemented modules over Dedekind domains. We prove that, an $R$-module $M$ is small supplemented if and only if every primary component of $T(M)$ is a direct sum of a bounded submodule and an artinian submodule, and $M / T(M)$ has finite uniform dimension, where $T(M)$ is the torsion submodule of $M$.

\section{Proper Classes}

Let us recall the definition of a proper class of short exact sequences (e.g., see [4], [7], [11], [19]).

2.1. Definition. Let $\mathcal{P}$ be a class of short exact sequences of $R$-modules and $R$-module homomorphisms. If a short exact sequence $\mathbb{E}: 0 \rightarrow A \stackrel{f}{\rightarrow} B \stackrel{g}{\rightarrow} C \rightarrow 0$ belongs to $\mathcal{P}$, then $f$ is said to be a $\mathcal{P}$-monomorphism and $g$ is said to be an $\mathcal{P}$-epimorphism. Also, $\mathbb{E}$ is said to be a $\mathcal{P}$-exact sequence.

The class $\mathcal{P}$ is said to be a proper class (in the sense of Buchsbaum) if it has the following properties:

$\mathrm{P}-1) \mathcal{P}$ is closed under isomorphisms;

$\mathrm{P}-2) \mathcal{P}$ contains all splitting short exact sequences;

$\mathrm{P}$-3) The class of $\mathcal{P}$-monomorphisms is closed under composition; if $f, g$ are monomorphisms and $g f$ is an $\mathcal{P}$-monomorphism, then $f$ is an $\mathcal{P}$-monomorphism;

$\mathrm{P}$-4) The class of $\mathcal{P}$-epimorphisms is closed under composition; if $f, g$ are epimorphisms and $g f$ is an $\mathcal{P}$-epimorphism, then $g$ is an $\mathcal{P}$-epimorphism.

2.2. Example. Some examples of proper classes, which are interesting for the purpose of this paper are the following (e.g., see [7]).

(i) The class Split of all splitting short exact sequences.

(ii) The class $\mathcal{P}$ of all short exact sequences on which the functor $\operatorname{Hom}(M,-)$ is exact for every $M \in \mathcal{M}$, where $\mathcal{M}$ is a class of modules. Its elements are called $\mathcal{P}$-pure exact sequences. For the class $\mathcal{M}$ of finitely presented modules, one has the classical pure exact sequences.

(iii) The classes of all short exact sequences $0 \rightarrow A \stackrel{f}{\rightarrow} B \rightarrow C \rightarrow 0$ with $\operatorname{Im} f$ is a supplement, or a closed submodule of $B$ are proper classes.

The set $\operatorname{Ext}_{\mathcal{P}}^{1}(C, A)$ of all short exact sequence of $\operatorname{Ext}_{R}^{1}(C, A)$ that belongs to a proper class $\mathcal{P}$ is a subgroup of the group of the extensions $\operatorname{Ext}_{R}^{1}(C, A)$. Conversely given a class $\mathcal{P}$ of short exact sequences if $\operatorname{Ext}_{\mathcal{P}}^{1}(C, A)$ is a subfunctor of $\operatorname{Ext}_{R}^{1}(C, A), \operatorname{Ext}_{\mathcal{P}}^{1}(C, A)$ is a subgroup of $\operatorname{Ext}_{R}^{1}(C, A)$ for every $R$-modules $A, C$ and the composition of two $\mathcal{P}_{-}$ monomorphisms (or $\mathcal{P}$-epimorphisms) is a $\mathcal{P}$-monomorphism (a $\mathcal{P}$-epimorphism respectively) then $\mathcal{P}$ is a proper class (see Theorem 1.1 in [14]). For any class $\mathcal{P}$ of short exact sequences the intersection $\langle\mathcal{P}\rangle$ of all proper classes containing $\mathcal{P}$ is clearly a proper class. We say that $\langle\mathcal{P}\rangle$ is the proper class generated by $\mathcal{P}$ (see [15]). Clearly $\langle\mathcal{P}\rangle$ is the least proper class containing $\mathcal{P}$.

2.3. Definition. [3] A short exact sequence $\mathbb{E}: 0 \rightarrow A \rightarrow B \rightarrow C \rightarrow 0$ is said to be extended weak supplement if there is a short exact sequence $\mathbb{E}^{\prime}: 0 \rightarrow A \stackrel{f}{\rightarrow} B^{\prime} \rightarrow C^{\prime} \rightarrow 0$ such that $\operatorname{Im} f$ has (is) a weak supplement in $B^{\prime}$ and there is a homomorphism $g: C \rightarrow C^{\prime}$ such that $\mathbb{E}=g^{*}\left(\mathbb{E}^{\prime}\right)$, that is, there is a commutative diagram as follows:

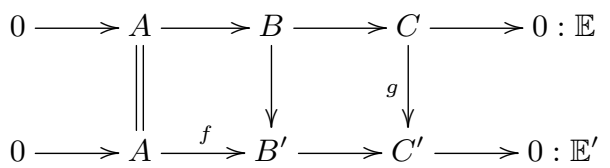


The class of all extended weak supplement short exact sequences will be denoted by $\overline{\mathcal{W S}}$. So $\operatorname{Ext}_{\overline{\mathcal{W S}}}(C, A)=\left\{\mathbb{E}: 0 \rightarrow A \rightarrow B \rightarrow C \rightarrow 0 \mid \mathbb{E}=g^{*}\left(\mathbb{E}^{\prime}\right)\right.$ for some $\mathbb{E}^{\prime}: 0 \rightarrow A \stackrel{f}{\rightarrow} B^{\prime} \rightarrow$ $C^{\prime} \rightarrow 0 \in \mathcal{W S}$ and $\left.g: C \rightarrow C^{\prime}\right\}$.

The class $\overline{\mathcal{W S}}$ is the least proper class containing the class $\mathcal{W} \mathcal{S}$ (see, [3]).

\section{The Proper Class $\mathcal{S} S$}

3.1. Definition. A submodule $L$ of an $R$-module $M$ has a small supplement in $M$ if there is a submodule $K$ of $M$ such that $L+K=M$ and $L \cap K$ is a small module.

Let $\mathcal{S} \mathcal{S}$ be the class of all short exact sequences $\mathbb{E}: 0 \rightarrow A \stackrel{f}{\rightarrow} B \rightarrow C \rightarrow 0$ such that $\operatorname{Im} f$ has a small supplement in $B$. To prove that $\mathcal{S} \mathcal{S}$ is a proper class we will use the result of $[14$, Theorem 1.1].

Firstly, we show that $\operatorname{Ext}_{s \mathcal{S}}(C, A)$ is a subgroup of $\operatorname{Ext}^{1}(C, A)$ for every $R$-modules $A, C$. The following lemma can be proved by using similar arguments as in $[3$, Lemma $3.3]$.

3.2. Lemma. For every homomorphism $f: A \rightarrow A^{\prime}, f_{*}: \operatorname{Ext}(C, A) \rightarrow \operatorname{Ext}\left(C, A^{\prime}\right)$ preserves short exact sequences from $\mathcal{S} S$.

3.3. Lemma. For every homomorphism $g: C^{\prime} \rightarrow C$, the homomorphism $g^{*}$ : $\operatorname{Ext}(C, A) \rightarrow \operatorname{Ext}\left(C^{\prime}, A\right)$ preserves short exact sequences from $\mathcal{S} \mathcal{S}$.

Proof. Let $\mathbb{E}: 0 \rightarrow A \rightarrow B \stackrel{h}{\rightarrow} C \rightarrow 0$ be a short exact sequence in $\mathcal{S} \mathcal{S}$ and $g: C^{\prime} \rightarrow C$ be a homomorphism. Then the following diagram is commutative with exact rows.

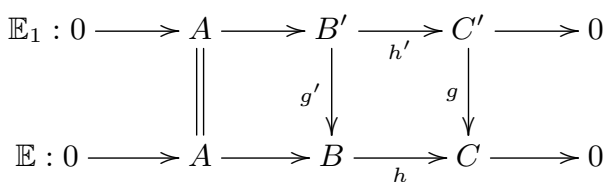

where $g^{*}(\mathbb{E})=\mathbb{E}_{1}$. Let $V$ be a small supplement of Ker $h$ in $B$. Then $\operatorname{Ker} h+V=B$ and $V \cap \operatorname{Ker} h$ is a small module. Then $g^{\prime-1}(V)+\operatorname{Ker} h^{\prime}=B^{\prime}$ by the pullback diagram. Since $g^{\prime}$ induces an isomorphism between $g^{\prime-1}(V) \cap \operatorname{Ker} h^{\prime}$ and $V \cap \operatorname{Ker} h, g^{\prime-1}(V) \cap \operatorname{Ker} h^{\prime}$ is a small module by [9, Theorem 2]. Therefore, $E_{1} \in \mathcal{S} \mathcal{S}$.

The proof of the following is routine, hence we skip its proof.

3.4. Proposition. If $\mathbb{E}_{1}, \mathbb{E}_{2} \in \operatorname{Ext}_{\mathcal{S}}(C, A)$, then $\mathbb{E}_{1} \oplus \mathbb{E}_{2} \in \operatorname{Ext}_{\mathcal{S}}(C \oplus C, A \oplus A)$.

3.5. Corollary. $\operatorname{Ext}_{\mathcal{~}}(C, A)$ is a subgroup of $\operatorname{Ext}(C, A)$ for every modules $C$ and $A$.

Proof. Let $\mathbb{E}_{1}, \mathbb{E}_{2} \in \operatorname{Extss}(C, A) . \mathbb{E}_{1} \oplus \mathbb{E}_{2}$ is $\mathcal{S} \mathcal{S}$-element by Proposition 3.4. Since $\mathbb{E}_{1}+\mathbb{E}_{2}=\nabla_{A}\left(\mathbb{E}_{1} \oplus \mathbb{E}_{2}\right) \Delta_{C}$ where $\Delta_{C}: c \mapsto(c, c)$ is the the diagonal map and $\nabla_{A}$ : $\left(a_{1}, a_{2}\right) \mapsto a_{1}+a_{2}$ is the codiagonal map, $\mathbb{E}_{1}+\mathbb{E}_{2}$ is in $\mathcal{S} \mathcal{S}$ by Lemma 3.2 and Lemma 3.3 .

3.6. Theorem. If $R$ is a left hereditary ring, then $\mathcal{S S}$ is a proper class.

Proof. By Lemma 3.2, Lemma 3.3, Corollary 3.5, $\operatorname{Ext}_{\mathcal{S} \mathcal{S}}^{1}(C, A)$ is a subfunctor of $\operatorname{Ext}_{R}^{1}(C, A)$ and $\operatorname{Ext}_{\mathcal{S} \mathcal{S}}^{1}(C, A)$ is a subgroup of $\operatorname{Ext}_{R}^{1}(C, A)$ for every $R$-modules $A$ and $C$. By [14, Theorem 1.1], we only need to show that the composition of two $\mathcal{S} S$-epimorphisms is an $\mathcal{S} S$-epimorphism. Let $f: B \rightarrow B^{\prime}$ and $g: B^{\prime} \rightarrow C$ be $\mathcal{S} \mathcal{S}$-epimorphisms. We have the following commutative diagram with exact rows and columns: 


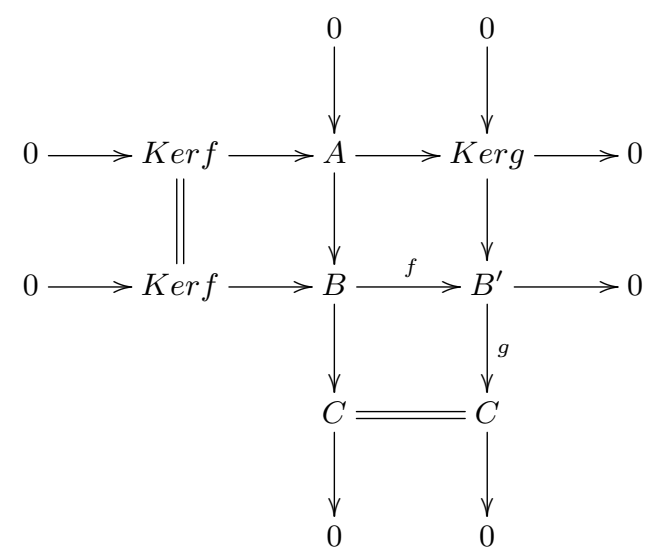

where $A / \operatorname{Ker} f \cong \operatorname{Ker} g, B / \operatorname{Ker} f \cong B^{\prime}$. Therefore there exist a submodule $V$ in $B$ such that $\operatorname{Ker} f+V=B$ and $\operatorname{Ker} f \cap V$ is a small module and there exist a submodule $U / \operatorname{Ker} f$ in $B / \operatorname{Ker} f$ such that $(U / \operatorname{Ker} f)+(A / \operatorname{Ker} f)=B / \operatorname{Ker} f$ and $(A \cap U) / \operatorname{Ker} f$ is a small module. By modular law, $A=\operatorname{Ker} f+(A \cap V), U=\operatorname{Ker} f+(U \cap V)$, $A \cap U=\operatorname{Ker} f+(A \cap V \cap U)$. Therefore, $B=A+U=A+(U \cap V)$ and $(A \cap U) / \operatorname{Ker} f \cong$ $(A \cap U \cap V) /(\operatorname{Ker} f \cap V)$. Since Ker $f \cap V$ and $(A \cap U \cap V) /(\operatorname{Ker} f \cap V)$ are small modules and $R$ is a hereditary ring, $A \cap U \cap V$ is small by [9, Theorem 3]. Hence $g \circ f$ is a SS-epimorphism.

A module $M$ is said to be $\overline{\mathcal{W S}}$-coinjective if every extension of $M$ is extended weak supplement.

3.7. Theorem. The classes $\mathcal{S} \mathcal{S}$ and $\overline{\mathcal{W} S}$ coincide over left hereditary rings.

Proof. Let $0 \rightarrow A \rightarrow B \rightarrow C \rightarrow 0 \in \mathcal{S}$ S. Then there is a submodule $V$ of $B$ such that $B=A+V$ and $A \cap V$ is a small module. So we have the following commutative diagram with exact columns and rows:

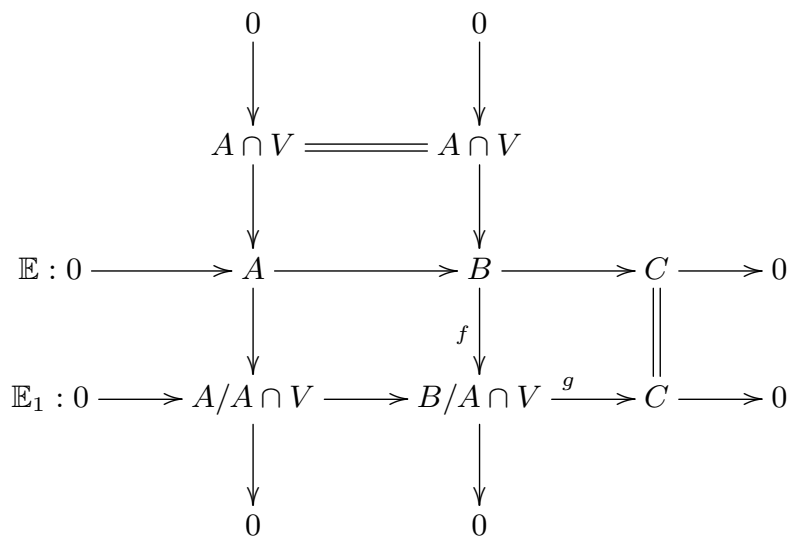

Clearly $g$ is a Split-epimorphism, and since small modules are $\overline{\mathcal{W S}}$-coinjective by $[3$, Theorem 4.1], $f$ is an $\overline{\mathcal{W S}}$-epimorphism. Since $R$ is hereditary, $\overline{\mathcal{W S}}$ is a proper class by [3, Theorem 3.12], and hence the composition $g \circ f$ is a $\overline{\mathcal{W S}}$-epimorphism. Then, $E$ is in $\overline{\mathcal{W S}}$. Conversely, since $\mathcal{W S} \subseteq \mathcal{S} \mathcal{S}$ and $\overline{\mathcal{W S}}$ is the smallest proper class containing $\mathcal{W} \mathcal{S}$, we have $\overline{\mathcal{W S}} \subseteq \mathcal{S} \mathcal{S}$. 
3.8. Lemma. The composition $g \circ f$ of a Split-epimorphism $f$ and an SS-epimorphism $g$ is an $\mathcal{S} S$-epimorphism.

Proof. Let $f: B \rightarrow B^{\prime}$ be a Split-epimorphism and $g: B^{\prime} \rightarrow C$ an SS-epimorphism. We have the following commutative diagram with exact rows and columns:

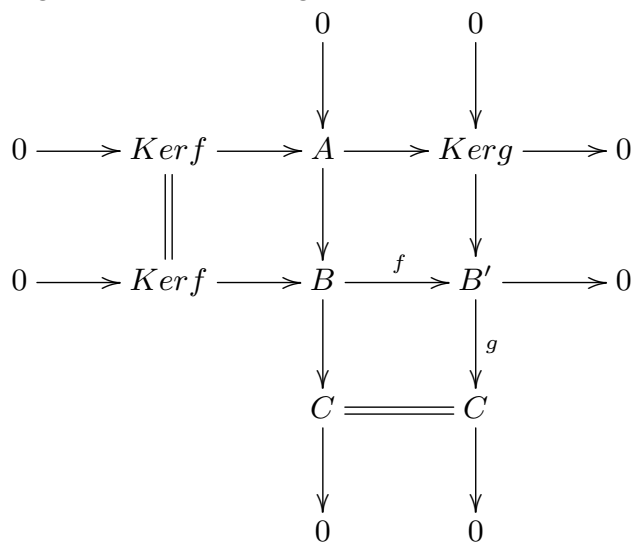

where $A / \operatorname{Ker} f \cong \operatorname{Ker} g, B / \operatorname{Ker} f \cong B^{\prime}$. Therefore there exist a submodule $V$ in $B$ such that $\operatorname{Ker} f \oplus V=B$ and there exist a submodule $U / \operatorname{Ker} f$ in $B / \operatorname{Ker} f$ such that $(U / \operatorname{Ker} f)+(A / \operatorname{Ker} f)=B / \operatorname{Ker} f$ and $(A \cap U) / \operatorname{Ker} f$ is a small module. By modular law, $U=\operatorname{Ker} f \oplus(U \cap V), A \cap U=\operatorname{Ker} f \oplus(A \cap V \cap U)$. Therefore, $B=A+U=A+(U \cap V)$. Since $(A \cap U) /$ Ker $f$ is a small module and $(A \cap U) / \operatorname{Ker} f \cong(A \cap U \cap V), A \cap U \cap V$ is a small module. Hence $g \circ f$ is an $\mathcal{S} \mathcal{S}$-epimorphism.

An epimorphism $f: N \rightarrow M$ is said to be a small cover of $M$ if $\operatorname{Ker} f \ll N$. Moreover, if $N$ is projective, then $f$ is called a projective cover. A ring $R$ is said to be left (semi) perfect if every (finitely generated) module has a projective cover.

3.9. Corollary. If $R$ is a left perfect ring, then every short exact sequence is an $\mathcal{S} S$-exact. In particular, $\mathcal{S} \mathcal{S}$ is a proper class.

Proof. Let $0 \rightarrow A \rightarrow B \stackrel{f}{\rightarrow} C \rightarrow 0$ be a short exact sequence. Since $R$ is left perfect ring, there exists an epimorphism $g: P \rightarrow C$ where $P$ is a projective $R$-module and Ker $g \ll P$. Therefore, $g$ is an $\mathcal{S} \mathcal{S}$-epimorphism. Consider the pullback diagram:

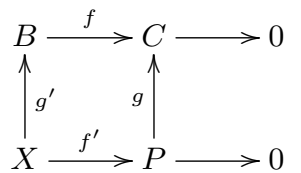

Since $P$ is projective, $f^{\prime}$ is a $\mathcal{S} p l i t$-epimorphism. Then $g \circ f^{\prime}$ is an $\mathcal{S} \mathcal{S}$-epimorphism by Lemma 3.8, and hence $f$ is an $\mathcal{S} \mathcal{S}$-epimorphism by $P-4$ ).

\section{Homological Objects of The Class $\mathcal{S} \mathcal{S}$}

We begin with the following definition.

4.1. Definition. An $R$-module $F$ is called $\mathcal{S} \mathcal{S}$-projective if it is projective relative to the short exact sequences that belong to $\mathcal{S} \mathcal{S}$ i.e., for each $\mathbb{E}$ in $\mathcal{S} \mathcal{S}$ the sequence $\operatorname{Hom}(F, \mathbb{E})$ is exact.

4.2. Proposition. The following are equivalent for an $R$-module $F$.

(1) $F$ is $\mathcal{S} \mathcal{S}$-projective. 
(2) $\operatorname{Ext}(F, S)=0$ for each small $R$-module $S$.

Proof. $(1) \Rightarrow(2)$ is clear, since every short exact sequence starting with a small module is in $\mathcal{S} \mathcal{S}$.

$(2) \Rightarrow(1)$ Let $0 \rightarrow A \rightarrow B \rightarrow C \rightarrow 0$ be a short exact sequence belongs to $\mathcal{S} \mathcal{S}$. Then there is a submodule $V$ of $B$ such that $B=A+V$ and $A \cap V$ is a small module. So we have the following commutative diagram

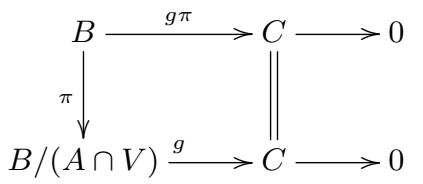

where $\pi$ is the canonical epimorphism and $g$ a split epimorphism. Applying the functor $\operatorname{Hom}(F,$.$) , we have the following diagram$

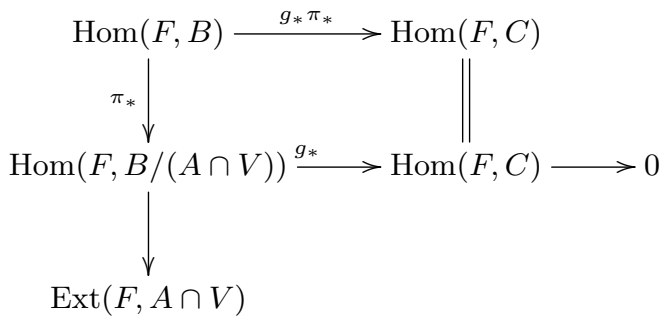

Since $g$ is a split epimorphism, $g_{*}$ is an epimorphism. Then $\operatorname{Ext}(F, A \cap V)=0$ by (2), and so $\pi_{*}$ is an epimorphism. Therefore, $g_{*} \pi_{*}$ is an epimorphism. Thus $F$ is an SS-projective module.

Note that every (finitely generated) $\mathcal{S} S$-projective module is projective if $R$ is left (semi) perfect by Proposition 4.2.

A ring $R$ is said to be left $C$-ring if $\operatorname{Soc}(R / I) \neq 0$ for each proper essential left ideal $I$ of $R$, (see [16]). Right perfect rings and left semiartinian rings are left $C$-rings. One of the characterization of left $C$-rings is the following: $R$ is a left $C$-ring if and only if $\operatorname{Ext}(S, M)=0$ for each simple $R$-module $S$ implies $M$ is injective $R$-module, ([20, Lemma $4])$.

4.3. Theorem. Let $R$ be a commutative $C$-ring. Then $\mathcal{S} \mathcal{S}$-projective $R$-modules are flat.

Proof. Let $M$ be an $\mathcal{S} \mathcal{S}$-projective $R$-module. Since every simple $R$-module is either small or injective, for each simple $R$-module $S, \operatorname{Ext}(M, S)=0$ by Proposition 4.2. Note that if $R$ is commutative and $E$ is an injective cogenerator, then $\operatorname{Hom}(S, E) \cong S$ for each simple $R$-module $S$. Then $\operatorname{Ext}(M, \operatorname{Hom}(S, \mathbb{Q} / \mathbb{Z})=0$. By the standart adjoint isomorphism $\operatorname{Hom}(\operatorname{Tor}(M, S), \mathbb{Q} / \mathbb{Z}) \cong \operatorname{Ext}(S, \operatorname{Hom}(M, \mathbb{Q} / \mathbb{Z}))=0$. Hence, $\operatorname{Hom}(\operatorname{Tor}(M, S), \mathbb{Q} / \mathbb{Z})=0$ by $[17$, Theorem 2.75]. But $R$ is $C$-ring, and $\operatorname{so} \operatorname{Hom}(M, \mathbb{Q} / \mathbb{Z})$ is injective. Therefore, $M$ is flat by [17, Proposition 3.54].

Renault [16] proved that a left Noetherian ring is a $C$-ring if and only if for every essential left ideal $I$ of $R, R / I$ has finite length. If $R$ is a left and right Noetherian, and left and right hereditary ring, then for every essential (proper) left ideal $I$ of $R$, the left $R$-module $R / I$ has finite length, ([12, Proposition 5.4.5 ]). Therefore, hereditary Noetherian commutative rings are $C$-rings. 
In [15], it is shown that, for a class of short exact sequences $\mathcal{E}$, modules which are relatively projective with respect to the classes $\mathcal{E}$ and $\langle\mathcal{E}\rangle$ coincide. Therefore, by Theorem 3.7, we get:

4.4. Corollary. Let $R$ be a commutative hereditary Noetherian ring. Then every $R$ module which is relatively projective with respect to the short exact sequences in $\mathcal{W S}$ is flat.

4.5. Remark. Let $M$ be a left $R$-module. $M$ is called $\mathcal{S} \mathcal{S}$-coinjective if every short exact sequence starting with $M$ is in $\mathcal{S} \mathcal{S}$. Every small module is $\mathcal{S} \mathcal{S}$-coinjective. $M$ is called almost injective if $M$ is a supplement submodule in each module that contains $M$ as a submodule see [6]. It is easy to see that almost injective modules are $\mathcal{S} \mathcal{S}$-coinjective, but the converse is not true in general. For example, $\mathbb{Z}$ is a small module, and so $\mathbb{Z}$ is $\mathcal{S S}$-coinjective. On the other hand, $\mathbb{Z}$ has no supplement in $\mathbb{Q}$, hence it is not almost injective.

Recall that a ring $R$ is called a left $V$-ring if every simple $R$-module is injective or, equivalently, $\operatorname{Rad}(M)=0$ for every $R$-module $M$ (see [8, Theorem 3.75]).

4.6. Proposition. The ring $R$ is a left $V$-ring if and only if every SS-coinjective $R$ module is injective.

Proof. Let $M$ be an $\mathcal{S} S$-coinjective $R$-module. Then $M$ is small supplement in $E(M)$ i.e., there is a submodule $V$ of $E(M)$ such that $E(M)=A+V$ and $A \cap V \ll E(M)$. But $R$ is $V$-ring, hence $A \cap V=0$. Then $A$ is direct summand of $E(M)$, and so it is injective. The converse follows easily since every simple $R$-module is either small or injective.

\section{Small Supplemented Modules}

An $R$-module $M$ is called small supplemented if every submodule of $M$ has a small supplement. In this section, we shall prove some properties of small supplemented modules. The proof of the following proposition is standard. We shall use it in the sequel.

5.1. Proposition. Let $M_{1}, U$ be submodules of $M$ with $M_{1}$ small supplemented. If there is a small supplement for $M_{1}+U$ in $M$, then $U$ also has a small supplement in $M$.

Proof. Let $V$ be a small supplement of $M_{1}+U$ in $M$, i.e. $V+\left(M_{1}+U\right)=M$ and $V \cap\left(M_{1}+U\right)$ is small. Since $M_{1}$ is small supplemented, there exist a submodule $T$ of $M_{1}$ such that $T+\left[M_{1} \cap(V+U)\right]=M_{1}$ and $T \cap\left[M_{1} \cap(V+U)\right]=T \cap(V+U)$ is a small module. Then $M=V+T+\left[M_{1} \cap(V+U)\right]+U=V+T+U$. Hence $U \cap(V+T) \subseteq T \cap(V+U)+V \cap(T+U)$, and so $U \cap(V+T)$ is a small module by [9, Theorem 2].

5.2. Corollary. If $M=M_{1}+M_{2}$ with $M_{1}, M_{2}$ small supplemented modules, then $M$ is also small supplemented.

Proof. For every submodule $N \subseteq M, M_{1}+\left(M_{2}+N\right)$ has the trivial small supplement and so, by Proposition 5.1, $M_{2}+N$ has a small supplement. Then, again by Proposition $5.1, N$ has a small supplement.

5.3. Proposition. The class of small supplemented modules is closed under submodules, homomorphic images and finite sums.

Proof. Let $L$ be a submodule of a small supplemented module $M$. Suppose $T \subseteq L$ and $N$ is a small supplement of $T$ in $M$. Then $L=T+(N \cap L)$ and $T \cap N \cap L \subseteq N \cap T \ll E(N \cap T)$ showing that $L$ is small supplemented. 
Let $N$ be a submodule of a small supplemented module $M$. Given a submodule $K / N$ of $M / N$, let $L$ be a small supplement of $K$ in $M$. Then $M=K+L$ and $K \cap L \ll E(K \cap L)$. Thus $K / N+(L+N) / N=M / N$ and $(K / N) \cap((L+N) / N)=((K \cap L)+N) / N \cong$ $(K \cap L) /(N \cap L)$ is a small module by [9, Theorem 2]. Thus $(L+N) / N$ is a small supplement of $K / N$ in $M / N$. So $M / N$ is small supplemented. The rest of the proof hold by Corollary 5.2 .

Note that small modules are closed under extensions over left hereditary rings (see, $[9])$.

5.4. Lemma. Let $R$ be a hereditary ring and $M$ be a small supplemented $R$-module. If $f: N \rightarrow M$ is an epimorphism with Ker $f$ a small module, then $N$ is small supplemented.

Proof. Let $K=\operatorname{Ker} f$. Since $M \cong N / K, N / K$ is small supplemented by Proposition 5.3. Let $L$ be a submodule of $N$. Then $(L+K) / K$ has a small supplement, say $T / K$, in $N / K$. So that $((L+K) / K)+(T / K)=N / K$ and $[(L+K) / K] \cap(T / K)=((T \cap L)+K) / K \cong$ $(T \cap L) /(K \cap L)$ is a small module. Then $N=L+T$ and $L \cap T$ is a small module by [9, Theorem 3]. Therefore $N$ is small supplemented.

5.5. Proposition. Let $R$ be a hereditary ring and $0 \rightarrow L \rightarrow M \rightarrow N \rightarrow 0$ be a short exact sequence from $\mathcal{S} S$. Then $L$ and $N$ are small supplemented if and only if $M$ is small supplemented.

Proof. Without restriction of generality we will assume that $L \subseteq M$. Let $S$ be a small supplement of $L$ in $M$ i.e. $L+S=M$ and $L \cap S \ll E(L \cap S)$. Then we have,

$$
M /(L \cap S)=L /(L \cap S) \oplus S /(L \cap S)
$$

$L /(L \cap S)$ is small supplemented as a factor module of $L$ by Proposition 5.3. On the other hand, $S /(L \cap S) \cong M / L \cong N$ is small supplemented. Then $M /(L \cap S)$ is small supplemented as a sum of small supplemented modules by Proposition 5.3. Therefore M is small supplemented by Lemma 5.4. The converse holds by Proposition 5.3.

A submodule $L \leq M$ is called coclosed in $M$, if for any proper submodule $K \leq L$, there is a submodule $N$ of $M$ such that $L+N=M$ but $K+N \neq M$. A module $\mathrm{M}$ is called weakly injective if for every extension $X$ of $M, M$ is coclosed in $X$. The properties of weakly injective modules are studied in [23].

5.6. Proposition. Let $M$ be a weakly injective module. Then $M$ is small supplemented if and only if $M$ is weakly supplemented.

Proof. $(\Rightarrow)$ Suppose $M$ is small supplemented and let $L$ be any submodule of $M$. Then $M=L+T$ and $L \cap T \ll E(M)$ where $T$ is small supplement of $L$ in $M$. Since $M$ is weakly injective module, it is coclosed in its injective hull $E(M)$ and so $L \cap T \ll M$ by [23, Lemma A.2].

$(\Leftarrow)$ Clear.

5.7. Proposition. Every $R$-module is small supplemented if and only if every injective $R$-module is weakly supplemented.

Proof. Suppose that $I$ is an injective $R$-module. Let $L$ be any submodule of $I$. By the assumption, there is a submodule $T$ of $I$ such that $I=L+T$ and $L \cap T \ll I$. Conversely, $E(M)$ is weakly supplemented for any $R$-module $M$ by the assumption. Then $M$ is small supplemented by Proposition 5.3. 


\section{Small Supplemented Modules Over Dedekind Domains}

In this section, we shall describe the structure of small supplemented modules over Dedekind domains. Recall that, a local Dedekind domain is called a discrete valuation ring (or, DVR). If $R$ is a DVR, then the unique maximal ideal of $R$ is of the form $p R$, for some $p \in R$ and every nonzero ideal of $R$ is of the form $p^{n} R$ for some $n \in \mathbb{Z}^{+}$. For a Dedekind domain $R, \Omega$ and $Q$ will stand for the set of maximal ideals of $R$, and the quotient ring of $R$ respectively.

A module $M$ is called coatomic, if $\operatorname{Rad}(M / N) \neq M / N$ for every proper submodule $N$ of $M$, equivalently every proper submodule of $M$ is contained in a maximal submodule, (see [22]). Recall that a module $M$ over a Dedekind domain is divisible if and only if it is injective if and only if it has no maximal submodules (see, [1], [18]).

The following lemma can be obtained from [22, Section 4]. We include it for completeness.

6.1. Lemma. Let $R$ be a Dedekind domain and $M$ be an $R$-module. Then $M$ is coatomic if and only if $M$ is a small module.

Proof. Let $M$ be a coatomic module and suppose $M+K=E(M)$ for some submodule $K$ of $M$. Then $M / M \cap K \cong E(M) / K$ is injective and so $E(M) / K$ has no maximal submodules. As $M$ is coatomic, we must have $M /(M \cap K)=0$, i.e. $M \subseteq K$. So that $K=E(M)$, and hence $M$ is a small module.

Conversely, if $M$ is small and $\operatorname{Rad}(M / K)=M / K$ for some $K \subseteq M$, then $M / K$ is injective. So that $M / K$ is a direct summand of $E(M) / K$. On the other hand $M / K$ is a small module as a factor of the small module $M$, a contradiction. Hence $K=M$ and so $M$ is coatomic.

6.2. Lemma. Let $R$ be a Dedekind domain and $M$ be a small supplemented $R$-module. Then $\operatorname{Rad}(M)$ has a weak supplement in $M$.

Proof. Since $M$ is small supplemented, $\operatorname{Rad}(M)+L=M$ and $\operatorname{Rad}(M) \cap L$ is a small module, for some $L \subseteq M$. Let $A=\operatorname{Rad}(M) \cap L$ and suppose that $A+Y=M$ for some $Y \varsubsetneqq M$. Then $A$ is coatomic by Lemma 6.1 , and so $A /(A \cap Y) \cong(A+Y) / Y=M / Y$ is also coatomic. So there is a maximal submodule $Z$ of $M$ containing $Y$. Now, we have $M=\operatorname{Rad}(M)+Y=\operatorname{Rad}(M)+Z \subseteq Z$, a contradiction. Therefore $A \ll M$, and so $L$ is a weak supplement of $\operatorname{Rad}(M)$ in $M$.

6.3. Lemma. [22, Lemma 4.1] Let $R$ be a commutative noetherian ring and $M$ be an $R$-module. Then a submodule $U$ of $M$ is small in $M$ if and only if $U_{\mathfrak{m}}$ is small in $M_{\mathfrak{m}}$ for every maximal ideal $\mathfrak{m}$ of $R$.

6.4. Lemma. Let $R$ be a commutative noetherian ring and $M$ be an $R$-module. If a submodule $V$ of $M$ is small supplement of a submodule $U$ of $M$, then $V_{\mathfrak{m}}$ is a small supplement of $U_{\mathfrak{m}}$ in $M_{\mathfrak{m}}$ for each maximal ideal $\mathfrak{m}$ of $R$.

Proof. Suppose $U+V=M$ and $U \cap V$ is a small module. Then $U_{\mathfrak{m}}+V_{\mathfrak{m}}=(U+V)_{\mathfrak{m}}=M_{\mathfrak{m}}$, and $(U \cap V)_{\mathfrak{m}}=U_{\mathfrak{m}} \cap V_{\mathfrak{m}}$ is a small module by Lemma 6.3. So that $V_{\mathfrak{m}}$ is a small supplement of $U_{\mathfrak{m}}$ in $M_{\mathfrak{m}}$.

6.5. Proposition. Let $R$ be a Dedekind domain and $M$ be a torsion free $R$-module. Then $M$ is small supplemented if and only if $M$ has finite uniform dimension.

Proof. Assume that the uniform dimension of $M$ is not finite. Then $M$ has a submodule $L$ such that $L \cong R^{(\mathbb{N})}$. Then $R^{(\mathbb{N})}$ is small supplemented by Proposition 5.3. Set $N=R^{(\mathbb{N})}$. 
Let $\mathfrak{m}$ be a maximal ideal of $R$. Then

$$
N_{\mathfrak{m}}=\left(R^{(\mathbb{N})}\right)_{\mathfrak{m}} \cong\left(R_{\mathfrak{m}}\right)^{(\mathbb{N})}
$$

and

$$
\operatorname{Rad}\left(N_{\mathfrak{m}}\right)=\mathfrak{m}\left((R)^{(\mathbb{N})}\right)_{\mathfrak{m}} \cong\left(\mathfrak{m} R_{\mathfrak{m}}\right)^{(\mathbb{N})}=\left(\mathfrak{m}_{\mathfrak{m}}\right)^{(\mathbb{N})}=\operatorname{Rad}\left(R_{\mathfrak{m}}\right)^{(\mathbb{N})}
$$

Now $(\mathfrak{m})^{(\mathbb{N})}$ has a small supplement in $R^{(\mathbb{N})}$. Then $\left(\mathfrak{m}_{\mathfrak{m}}\right)^{(\mathbb{N})}$ has a weak supplement in $\left(R_{\mathfrak{m}}\right)^{(\mathbb{N})}$ by Lemma 6.2 Lemma 6.3 and Lemma 6.4 . Therefore $R_{\mathfrak{m}}$ is a perfect ring by [5, Theorem 1]. This contradicts with the fact that $R_{\mathfrak{m}}$ is a domain. Therefore $M$ has a finite uniform dimension. Conversely, suppose $M$ has finite uniform dimension. Then $E(M) \cong Q^{n}$, where $Q$ is the quotient ring of $R$ and $n \in \mathbb{Z}^{+}$. Then $E(M)$ is weakly supplemented by [2, Lemma 2.8] and [10, Proposition 2.5]. So that $E(M)$ is small supplemented and so $M$ is small supplemented by Proposition 5.3.

6.6. Lemma. Let $R$ be a $D V R$ and $M$ be a torsion and reduced $R$-module. Then $M$ is small supplemented if and only if $M$ is bounded.

Proof. Suppose $M$ is small supplemented. Then $\operatorname{Rad}(M)=p M$ has a weak supplement by Lemma 6.2. Hence $L+p M=M$ and $L \cap p M$ is small for some $L \subseteq M$. Since $\frac{L}{L \cap p M} \cong \frac{L+p M}{p M}=\frac{M}{p M}$ is semisimple, it is coatomic. So that, $L$ is coatomic by [21, Lemma 1.5]. Then $L$ is bounded by [21, Lemma 2.1], that is, $p^{n} L=0$ for some $n \in \mathbb{Z}^{+}$. Hence we get $p^{n} M=p^{n}(p M+L)=p^{n+1} M=p\left(p^{n} M\right)$, and so $p^{n} M$ is divisible by [1, Lemma 4.4]. But $M$ is reduced, so that we must have $p^{n} M=0$.

The converse is clear, because bounded modules are small and small modules are small supplemented.

6.7. Lemma. Let $R$ be a DVR and $M$ be a divisible(injective) and torsion $R$-module. Then $M$ is small supplemented if and only if $M \cong(Q / R)^{n}$, for some $n \in \mathbb{Z}^{+}$.

Proof. Since $M$ is divisible and torsion, $M \cong(Q / R)^{(I)}$ for some index set $I$. Suppose $M$ is small supplemented. If $I$ is finite then we are done. Otherwise, $M$ has a submodule which is isomorphic to $L=\oplus_{i=1}^{\infty}<\frac{1}{p^{i}}+R>$. Then $L$ is small supplemented by Proposition 5.3 and so $L$ is bounded by Lemma 6.6, a contradiction. Hence $I$ is finite.

Conversely, if $M \cong(Q / R)^{n}$, then $M$ is weakly supplemented by [2, Lemma 2.8] and [10], and so $M$ is small supplemented.

6.8. Theorem. Let $R$ be a Dedekind domain and $M$ be a torsion $R$-module. Then $M$ is small supplemented if and only if $T_{P}(M)$ is small supplemented for every $P \in \Omega$.

Proof. $(\Rightarrow)$ Since $M$ is torsion, $M=\oplus_{P \in \Omega} T_{P}(M)$. Then $T_{P}(M)$ is small supplemented by Proposition 5.3.

$(\Leftarrow)$ Let $N$ be a submodule of $M$. As $M$ is a torsion module, $N=\oplus_{P \in \Omega} N_{P}$, where $N_{P}=N \cap T_{P}(M)$. Let $K_{P}$ be a small supplement of $N_{P}$ in $T_{P}(M)$. Then it is straightforward to check that, for the submodule $K=\oplus_{p \in \Omega} K_{p}$, we have $N+K=M$ and $N \cap K$ is a small module. That is, $K$ is a small supplement of $N$. Hence $M$ is small supplemented.

6.9. Lemma. Let $R$ be a Dedekind domain and $M$ be an $R$-module. If $T(M)$ is small supplemented then $T(M)$ has a small supplement in $M$.

Proof. Let $T(M)=N \oplus D$, where $N$ is the reduced part and $D$ is the divisible part of $T(M)$. Write $N=\oplus_{P \in \Omega} T_{P}(N)$. Since $T(M)$ is small supplemented $N$ is small supplemented by Proposition 5.3. So that $T_{P}(N)$ is bounded, and so $T_{P}(N)$ is small in $E(N)$. Hence $N=\oplus_{P \in \Omega} T_{P}(N)$ is small in $E(N)$. Now as $N$ is small in $E(M)$ and $D$ 
is an injective module, $N$ and $D$ have small supplements in $E(M)$. If $E(M)=D \oplus D^{\prime}$, then $T(M)=D \oplus T(M) \cap D^{\prime}$. So that $N \cong T(M) \cap D^{\prime}$ is small, and hence $D^{\prime}$ is a small supplement of $T(M)$ in $E(M)$. Then $D^{\prime} \cap M$ is small supplement of $T(M)$ in $M$. This completes the proof.

6.10. Corollary. Let $R$ be a Dedekind domain and $M$ be an $R$-module. Then $M$ is small supplemented if and only if $T(M)$ and $M / T(M)$ are small supplemented.

Proof. $(\Rightarrow)$ By Proposition 5.3.

$(\Leftarrow)$ By Proposition 5.5 and Lemma 6.9.

Summing up, Lemma 6.6, Lemma 6.7, Theorem 6.8 and Corollary 6.10, we get:

6.11. Corollary. Let $R$ be a Dedekind domain and $M$ be an $R$-module. Then $M$ is small supplemented if and only if

(1) $M / T(M)$ has finite uniform dimension.

(2) For every $P \in \Omega$, the reduced part of $T_{P}(M)$ is bounded and the divisible part has finite uniform dimension.

We finish the paper by showing that every small supplemented module is $\mathcal{S} S$-coinjective over Dedekind domains. Recall that, every module $M$ over a Dedekind domain can be written as $M=N \oplus D$, where $D$ is divisible (equivalently, injective) and $N$ is reduced. Since injective modules are coinjective, $M$ is $\mathcal{S} \mathcal{S}$-coinjective if and only if $N$ is $\mathcal{S S}$-coinjective.

6.12. Theorem. Over a Dedekind domain $R$, every small supplemented $R$-module is $\mathcal{S}$-coinjective.

Proof. Let $M$ be a small supplemented module. Without loss of generality we may assume that $M$ is a reduced $R$-module. We shall prove that both $T(M)$ and $M / T(M)$ are $\mathcal{S} \mathcal{S}$-coinjective. Since $M$ is reduced and small supplemented, in the decomposition $T(M)=\oplus_{P \in \Omega} T_{P}(M)$ each $T_{P}(M)$ is bounded by Corollary 6.11. Every bounded module is small and so $T(M)$ is a small module by Lemma 6.3. Therefore $T(M)$ is $\mathcal{S} \mathcal{S}$-coinjective by [3, Theorem 4.1]. On the other hand, $M / T(M)$ has finite uniform dimension by Corollary 6.11. Then $M / T(M)$ is $\mathcal{S} \mathcal{S}$-coinjective by [3, Corollary 4.4]. By [13], coinjective modules are closed under extensions. Hence $M$ is $\mathcal{S} \mathcal{S}$-coinjective, as $T(M)$ and $M / T(M)$ both are $\mathcal{S} S$-coinjective.

\section{References}

[1] Alizade, R. and Bilhan, G. and Smith, P. F., Modules whose maximal submodules have supplements, Comm. Algebra, 29 (6), 2389-2405, 2001.

[2] Alizade, R. and Büyükaşik, E., Extensions of weakly supplemented modules, Math. Scand., 103 (2), 161-168, 2008.

[3] Alizade, R. and Demirci, Y. and Durgun, Y. and Pusat, D., The Proper Class Generated by Weak Supplements, Comm. Algebra, 42, 56-72, 2014.

[4] Buchsbaum, D. A., A note on homology in categories, Ann. of Math., 69, 66-74, 1959.

[5] Büyükaşik, E. and Lomp, C., Rings whose modules are weakly supplemented are perfect. Applications to certain ring extensions, Math. Scand., 105 (1), 25-30, 2009.

[6] Clark, J. and Tütüncü, D. K. and Tribak, R., Almost injective modules, Comm. Algebra, 39, 4390-4402, 2011.

[7] Clark, J. and Lomp, C. and Vanaja, N. and Wisbauer, R., Lifting modules, Frontiers in Mathematics, Basel, 2006.

[8] Lam, T. Y., Lectures on Modules and Rings, New York: Springer-Verlag, 1998.

[9] Leonard, W. W., Small modules, Proc. Amer. Math. Soc., 17, 527-531, 1966.

[10] Lomp, C., On semilocal modules and rings, Comm. Algebra, 27 (4), 1921-1935, 1999. 
[11] Mac Lane, S., Homology, Academic Press Inc., New York, 1963.

[12] McConnell, J. C. and Robson, J. C., Noncommutative Noetherian rings, John Wiley \& Sons Ltd., New York, 1987.

[13] Mishina, A. P. and Skornyakov, L. A., Abelian groups and modules, American Mathematical Society, 1976.

[14] Nunke, R. J., Purity and subfunctors of the identity, Topics in Abelian Groups (Proc. Sympos., New Mexico State Univ., Scott, Foresman and Co., Chicago, Ill., 121-171, 1963.

[15] Pancar, A., Generation of proper classes of short exact sequences, Internat. J. Math. Math. Sci., 20 (3), 465-473, 1997.

[16] Renault, G., Étude de certains anneaux A liés aux sous-modules compléments d'un Amodule, C. R. Acad. Sci. Paris 259, 4203-4205, 1964.

[17] Rotman, J. J., An introduction to homological algebra, Springer, New York, 2009.

[18] Sharpe, D. W. and Vámos, P., Injective modules, Cambridge University Press, London, 1972.

[19] Skljarenko, E. G., Relative homological algebra in the category of modules, Uspehi Mat. Nauk, 33 (3), 85-120, 1978.

[20] Smith, P. F., Injective modules and prime ideals, Comm. Algebra, 9 (3), 989-999, 1981.

[21] Zöschinger, H. Komplementierte Moduln über Dedekindringen, J. Algebra, 29, 42-56, 1974.

[22] Zöschinger, H. Koatomare Moduln, Math. Z., 170 (3), 221-232, 1980.

[23] Zöschinger, H. Schwach-injektive Moduln, Period. Math. Hungar., 52 (2), 105-128, 2006. 
